\title{
Quantum and pseudoclassical descriptions of nonrelativistic spinning particles in noncommutative space
}

\author{
T. C. Adorno*, M. C. Baldiotti ${ }^{\dagger}$ and D. M. Gitman ${ }^{\ddagger}$ \\ Instituto de Física, Universidade de São Paulo, \\ Caixa Postal 66318, CEP 05508-090, São Paulo, S.P., Brazil
}

September 17, 2018

\begin{abstract}
We construct a nonrelativistic wave equation for spinning particles in the noncommutative space (in a sense, a $\theta$-modification of the Pauli equation). To this end, we consider the nonrelativistic limit of the $\theta$-modified Dirac equation. To complete the consideration, we present a pseudoclassical model (à la Berezin-Marinov) for the corresponding nonrelativistic particle in the noncommutative space. To justify the latter model, we demonstrate that its quantization leads to the $\theta$-modified Pauli equation. We extract $\theta$-modified interaction between a nonrelativistic spin and a magnetic field from such a Pauli equation and construct a $\theta$-modification of the Heisenberg model for two coupled spins placed in an external magnetic field. In the framework of such a model, we calculate the probability transition between two orthogonal EPR (EinsteinPodolsky-Rosen) states for a pair of spins in an oscillatory magnetic field and show that some of such transitions, which are forbidden in the commutative space, are possible due to the space noncommutativity. This allows us to estimate an upper bound on the noncommtativity parameter.
\end{abstract}

*adorno@dfn.if.usp.br

†baldiott@fma.if.usp.br

†'gitman@dfn.if.usp.br 


\section{Introduction}

It is known that QFT's in noncommutative spaces induce the so-called $\theta$-modified relativistic wave equations, which, at present, are interpreted as Schrödinger equations of relativistic quantum mechanics in noncommutative spaces, see e.g., [1, 2, 3, 4, 5, 6, 7, 8, . Calculations in the framework of such theories allow one to evaluate the influence of space noncommutativity on different physical effects and, e.g., to establish upper bonds on the noncommutativity parameters $\theta$, see [9, 10, 11, 12]. In the present article, we construct a nonrelativistic wave equation for spinning particles in the noncommutative space (in a sense, a $\theta$-modification of the Pauli equation). In course of solving the above mentioned problem, we consider the nonrelativistic limit of the $\theta$-modified Dirac equation. It should be noted that the form of the latter equation depend essentially on the point of view how to write the action of the spinor field in an external background in the noncommutative space. We consider two possible actions, one obtained from simple Moyal modification [13] (introducing Moyal products into the ordinary Dirac field action [1, 2, 15, 14, 16, 17, 18, 19, 11, 20]) and another action obtained by the so-called Seiberg-Witten (SW) map, see [21, 22, 23, 24, 25, 26].

It should be noted that the noncommutativity can be also justified by a group theoretical analysis of the Galilean symmetry in nonrelativistic particle systems. A relation between spin and noncommutativity can be studied on examples of classical models of nonrelativistic spinning particles, considered in [27, 28], 29] and [30]. The corresponding quantum versions of the models describe anyons and their interaction with external fields in the relativistic and nonrelativistic context [31, 32].

Doing nonrelativistic limits in both $\theta$-modified Dirac equations, we follow the standard approach, see e.g. [33, 34], separating "big" and "small" two-component spinors in the Dirac four-component wave function. The equation for the big spinor is just the nonrelativistic wave equation for spinning particles. To complete the consideration, we present a pseudoclassical model (à la Berezin-Marinov [36, 37]) for the corresponding nonrelativistic particle in the noncommutative space. To justify the model, we demonstrate that its quantization leads to the $\theta$-modified wave equation for nonrelativistic spinning particle.

Then, we consider one of possible applications of the obtained general result. Namely, we extract $\theta$-modified interaction between a nonrelativistic spin and a magnetic field from the $\theta$-modified Pauli equation. With such an interaction in hands, we construct a $\theta$-modification of the Heisenberg model for two coupled spins placed in an external magnetic field. In the framework of such a model, we calculate the probability transition between two orthogonal EPR (Einstein-Podolsky-Rosen) states for a pair of spins in an oscillatory magnetic field and show that some of such transitions, which are forbidden in the commutative space, are possible due to the space noncommutativity. 


\section{Nonrelativistic limit of $\theta$-modified Dirac equation}

\subsection{Simple Moyal modification}

Here, we construct an action of the spinor field $\Psi$ (in an external electromagnetic background $\left.A^{\mu}\right)$ in a noncommutative (NC) space, introducing the Moyal star product in the ordinary spinor field action (we call this a simple Moyal modification in what follows). In such a way, we derive the following action 1

$$
\begin{aligned}
& S_{\mathrm{M}}^{\theta}=\int d x \mathcal{L}_{\mathrm{M}}^{\theta}, \quad \mathcal{L}_{\mathrm{M}}^{\theta}=\bar{\Psi}(x) \star\left(\gamma^{\mu} \hat{P}_{\mu}-m c\right) \star \Psi(x), \\
& \hat{P}_{\mu}(x)=\hat{p}_{\mu}-\frac{e}{c} A_{\mu}(x), \quad \hat{p}_{\mu}=i \hbar \partial_{\mu}, \quad A^{\mu}=\left(A^{0}, \mathbf{A}\right), \quad \mathbf{A}=\left(A^{i}, i=1,2,3\right),
\end{aligned}
$$

where the Moyal star product " $\star$ " is defined as

$$
f(x) \star g(x)=f(x) e^{\frac{i}{2} \overleftarrow{\partial}_{\mu} \theta^{\mu \nu} \vec{\partial}_{\nu}} g(x),
$$

with $f(x)$ and $g(x)$ being arbitrary functions, see e.g. [1, 2, 16, 17, 18, 19, 11, 20], and $x=\left(x^{0}=c t, x^{i}, \quad i=1,2,3\right)$. Here and in what follows, the subindex M reminds us that we use the simple Moyal modification.

Then the $\theta$-modified Dirac equation with an external electromagnetic field, for a particle with the charge $e$ (for an electron $e=-|e|$ ) and the mass $m$ is identified with Euler-Lagrange equation $\delta S_{\mathrm{M}}^{\theta} / \delta \bar{\Psi}=0$. Thus, we obtain:

$$
\begin{aligned}
& \left(\gamma^{\mu} \hat{P}_{\mu}^{\theta}-m c\right) \Psi(x)=0, \\
& \hat{P}_{\mu}^{\theta}=\hat{p}_{\mu}-\frac{e}{c} A_{\mu}\left(x^{\mu}+\frac{i}{2} \theta^{\mu \nu} \partial_{\nu}\right),
\end{aligned}
$$

where $\gamma^{\mu}=\left(\gamma^{0}, \gamma\right)$ are Dirac gamma-matrices, see, e.g. [1, 2].

As was mentioned above, our aim is to derive a nonrelativistic quantum mechanical description of a spinning particle in a noncommutative space. Since in the nonrelativistic case, time and the configuration space are considered separately, it is consistent (and natural) to treat space and space-time noncommutativity effects separately. In what follows, we consider the case of the space noncommutativity only, which implies $\theta^{0 \mu}=0$. Such a choice is also supported by the fact that in the framework of noncommutative classical or quantum mechanics, there exist physical motivations just for the space noncommutativity. In particular, the parameters $\theta^{i j}$ admit many close analogies with a constant magnetic field both from the algebraic and dynamical points of view [7, 8].

\footnotetext{
${ }^{1}$ By bold, we denote three-vectors, e.g., $\mathbf{a}=\left(a^{i}=-a_{i}, i=1,2,3\right)$.
} 
We can rewrite (2) in the Hamiltonian form (see, e.g. [1, 2, 9]),

$$
\begin{aligned}
& i \hbar \partial_{t} \Psi(x)=\widehat{\mathbb{H}}_{\mathrm{D}}(\hat{q}, \hat{\mathbf{p}}) \Psi(x), \quad \partial_{t}=\frac{\partial}{\partial t}, \\
& \widehat{\mathbb{H}}_{\mathrm{D}}(\hat{q}, \hat{\mathbf{p}})=c \boldsymbol{\alpha} \cdot \hat{\mathbf{P}}(\hat{q})+m c^{2} \gamma^{0}+e A^{0}(\hat{q}),
\end{aligned}
$$

where $\Psi(x)$, is a bispinor, $\hat{\mathbf{P}}(\hat{q})=\hat{\mathbf{p}}-\frac{e}{c} \mathbf{A}(\hat{q}), \hat{q}=\hat{q}^{\mu}=\left(x^{0}, \hat{q}^{i}\right)$,

$$
\begin{aligned}
& \hat{q}^{i}=x^{i}-\frac{1}{2 \hbar} \theta^{i j} \hat{p}^{j}, \quad \hat{p}^{j}=-i \hbar \partial_{j}, \\
& {\left[\hat{q}^{i}, \hat{q}^{j}\right]=i \theta^{i j}, \quad\left[\hat{q}^{i}, \hat{p}^{j}\right]=i \hbar \delta^{i j}, \quad\left[\hat{p}^{i}, \hat{p}^{j}\right]=0 .}
\end{aligned}
$$

In the first order in $\theta$, equation (2) reads:

$$
\left\{\gamma^{\mu}\left(\hat{P}_{\mu}(x)-\frac{i e}{2 c} \partial_{\alpha} A_{\mu}(x) \theta^{\alpha \beta} \partial_{\beta}\right)-m c\right\} \Psi(x)=0 .
$$

Being written in the Hamiltonian form, equation (5) reads:

$$
\begin{aligned}
& i \hbar \partial_{t} \Psi(x)=\hat{\mathbb{H}}_{\mathrm{M}}^{\theta} \Psi(x), \hat{\mathbb{H}}_{\mathrm{M}}^{\theta}=\hat{\mathbb{H}}_{\mathrm{D}}+\Delta \hat{\mathbb{H}}_{\mathrm{M}}^{\theta}, \\
& \hat{\mathbb{H}}_{\mathrm{D}}=c \boldsymbol{\alpha} \cdot \hat{\mathbf{P}}+e A_{0}+m c^{2} \gamma^{0}, \\
& \Delta \hat{\mathbb{H}}_{\mathrm{M}}^{\theta}=\frac{e}{2 \hbar}\left[\boldsymbol{\nabla}\left(\boldsymbol{\alpha} \cdot \mathbf{A}-A_{0}\right) \times \hat{\mathbf{p}}\right] \cdot \boldsymbol{\theta}, \\
& \hat{\mathbf{P}}=\hat{\mathbf{p}}-\frac{e}{c} \mathbf{A}, \quad \boldsymbol{\theta}=\left(\theta^{i}=\frac{1}{2} \varepsilon_{i j k} \theta^{j k}\right), \quad \boldsymbol{\alpha}=\gamma^{0} \boldsymbol{\gamma} .
\end{aligned}
$$

Let us consider the nonrelativistic limit of the latter equation following the standard scheme and doing transformations à la Foldy-Wouthuysen, see e.g. [33, 34. The Hamiltonian $\hat{\mathbb{H}}_{M}^{\theta}$ (6) is written in terms of an odd operaton $2 \mathcal{O}_{\mathrm{M}}=c \boldsymbol{\alpha} \cdot \hat{\mathbf{P}}+(e / 2 \hbar)[\boldsymbol{\nabla}(\boldsymbol{\alpha} \cdot \mathbf{A}) \times \hat{\mathbf{p}}] \cdot \boldsymbol{\theta}$ and an even operator $\mathcal{E}_{\mathrm{M}}=m c^{2} \gamma^{0}+e A_{0}-(e / 2 \hbar)\left[\boldsymbol{\nabla} A_{0} \times \hat{\mathbf{p}}\right] \cdot \boldsymbol{\theta}$. First, we perform the canonical transformation $\Psi^{(1)}=e^{i \hat{S}_{\mathrm{M}}^{(1)}} \Psi$, with $\hat{S}_{\mathrm{M}}^{(1)}=\left(2 i m c^{2}\right)^{-1} \gamma^{0} \mathcal{O}_{\mathrm{M}}$, trying to eliminate odd operators from $\hat{\mathbb{H}}_{\mathrm{M}}^{\theta}$ (deriving the nonrelativistic approximation, we neglected terms of the order $O\left(\left(m c^{2}\right)^{-3}\right)$ independent of $\left.\theta\right)$,

$$
i \hbar \partial_{t} \Psi^{(1)}=\hat{\mathbb{H}}_{\mathrm{M}}^{\theta(1)} \Psi^{(1)}, \quad \hat{\mathbb{H}}_{\mathrm{M}}^{\theta(1)}=e^{i \hat{S}_{\mathrm{M}}^{(1)}}\left(\hat{\mathbb{H}}_{\mathrm{M}}^{\theta}-i \hbar \partial_{t}\right) e^{-i \hat{S}_{\mathrm{M}}^{(1)}}
$$

\footnotetext{
${ }^{2}$ An operator which has only matrix elements connecting the upper and lower components of the Dirac spinor is classified as odd $\mathcal{O}$ and an operator which does not have such elements is classified as even $\mathcal{E}$.
} 
For $\hat{\mathbb{H}}_{\mathrm{M}}^{\theta(1)}$, we obtain

$$
\begin{aligned}
& \hat{\mathbb{H}}_{\mathrm{M}}^{\theta(1)}=\mathcal{E}_{\mathrm{M}}+\mathcal{E}_{\mathrm{M}}^{(1)}+\mathcal{O}_{\mathrm{M}}^{(1)}+\mathcal{O}_{\mathrm{M}}^{(1)}, \quad \mathcal{E}_{\mathrm{M}}^{(1)}=\frac{1}{2 m c^{2}} \gamma^{0} \mathcal{O}_{\mathrm{M}}^{2} \\
& -\frac{1}{8 m^{2} c^{4}}\left[\mathcal{O}_{\mathrm{M}},\left(e\left[\mathcal{O}_{\mathrm{M}},\left(A_{0}-\frac{1}{2 \hbar}\left[\boldsymbol{\nabla} A_{0} \times \hat{\mathbf{p}}\right] \cdot \boldsymbol{\theta}\right)\right]+i \hbar \partial_{t} \mathcal{O}_{\mathrm{M}}\right)\right] \\
& \mathcal{O}_{\mathrm{M}}^{(1)}=\frac{\gamma^{0}}{2 m c^{2}}\left(e\left[\mathcal{O}_{\mathrm{M}}, A_{0}\right]+i \hbar \partial_{t} \mathcal{O}_{\mathrm{M}}-\frac{e}{2 \hbar} \varepsilon_{i j k}\left[\mathcal{O}_{\mathrm{M}},\left(\partial_{i} A_{0}\right) \hat{p}^{j}\right] \theta^{k}\right) \\
& \mathcal{O}_{\mathrm{M}}^{\prime(1)}=-\frac{1}{3 m^{2} c^{4}} \mathcal{O}_{\mathrm{M}}^{3} .
\end{aligned}
$$

The first canonical transformation does not eliminate odd operators of the order $\left(m c^{2}\right)^{-1}$, that is why we need to perform a second canonical transformation with the generator $\hat{S}_{\mathrm{M}}^{(2)}=$ $\left(2 i m c^{2}\right)^{-1} \gamma^{0} \mathcal{O}_{\mathrm{M}}^{(1)}$. Thus, we obtain a Schrödinger equation with the Hamiltonian

$$
\begin{aligned}
& \hat{\mathbb{H}}_{\mathrm{M}}^{\theta(2)}=e^{i \hat{S}_{\mathrm{M}}^{(2)}}\left(\hat{\mathbb{H}}_{\mathrm{M}}^{\theta(1)}-i \hbar \partial_{t}\right) e^{-i \hat{S}_{\mathrm{M}}^{(2)}}=\mathcal{E}_{\mathrm{M}}+\mathcal{E}_{\mathrm{M}}^{(1)}+\mathcal{O}_{\mathrm{M}}^{(2)} \\
& \mathcal{O}_{\mathrm{M}}^{(2)}=\frac{e}{2 m c^{2}} \gamma^{0}\left(\left[\mathcal{O}_{\mathrm{M}}^{(1)}, A_{0}\right]-\frac{1}{2 \hbar} \varepsilon_{i j k}\left[\mathcal{O}_{\mathrm{M}}^{(1)},\left(\partial_{i} A_{0}\right) \hat{p}^{j}\right] \theta^{k}\right)+\mathcal{O}_{\mathrm{M}}^{(1)}-\hbar \partial_{t} \hat{S}_{\mathrm{M}}^{(2)} .
\end{aligned}
$$

The operator $\hat{\mathbb{H}}_{\mathrm{M}}^{\theta(2)}$ still contains the odd operator $\mathcal{O}_{\mathrm{M}}^{(2)}$ of the order $\left(m c^{2}\right)^{-2}$, the latter operator can be eliminated by a third canonical transformation with the generator $\hat{S}_{\mathrm{M}}^{(3)}=$ $\left(2 i m c^{2}\right)^{-1} \gamma^{0} \mathcal{O}_{\mathrm{M}}^{(2)}$. Finally, we get the Schrödinger equation with the Hamiltonian $\hat{\mathbb{H}}_{\mathrm{M}}^{\theta(3)}$ and the wave function $\Psi^{(3)}$. The new Hamiltonian $\hat{\mathbb{H}}_{\mathrm{M}}^{\theta(3)}$ is already an even operator, it has the form

$$
\hat{\mathbb{H}}_{\mathrm{M}}^{\theta(3)}=e^{i \hat{S}_{\mathrm{M}}^{(3)}}\left(\hat{\mathbb{H}}_{\mathrm{M}}^{\theta(2)}-i \hbar \partial_{t}\right) e^{-i \hat{S}_{\mathrm{M}}^{(3)}}=\mathcal{E}_{\mathrm{M}}+\mathcal{E}_{\mathrm{M}}^{(1)}=\operatorname{diag}\left(m c^{2}+\hat{H}_{\mathrm{M}}^{\theta}, \hat{h}^{\theta}\right)
$$

and

$$
\Psi^{(3)}=e^{i \hat{S}_{\mathrm{M}}^{(3)}} e^{i \hat{S}_{\mathrm{M}}^{(2)}} e^{i \hat{S}_{\mathrm{M}}^{(1)}} \Psi=\left(\begin{array}{c}
\psi(x) \\
\chi(x) \sim\left(m c^{2}\right)^{-1} \psi(x)
\end{array}\right) .
$$

In the approximation under consideration, equations for upper "big" bispinor $\psi$ and inferior "small" bispinor $\chi$ are independent. We interpret $\psi$ as the wave functions of the nonrelativistic spinning particle with the Hamiltonian $\hat{H}_{\mathrm{M}}^{\theta}$.

Retaining only terms of the order $\left(m c^{2}\right)^{-1}$ in $\hat{H}_{\mathrm{M}}^{\theta}(10)$, we obtain the following equation for $\psi$ :

$$
\begin{aligned}
& i \hbar \partial_{t} \psi(x)=\hat{H}_{\mathrm{M}}^{\theta} \psi(x), \\
& \hat{H}_{\mathrm{M}}^{\theta}=\frac{1}{2 m c^{2}} \mathcal{O}_{\mathrm{M}}^{2}+e A_{0}-\frac{e}{2 \hbar}\left[\boldsymbol{\nabla} A_{0} \times \hat{\mathbf{p}}\right] \cdot \boldsymbol{\theta},
\end{aligned}
$$

where

$$
\mathcal{O}_{\mathrm{M}}=c \boldsymbol{\alpha} \cdot \hat{\mathbf{P}}+\frac{e}{2 \hbar}[\nabla(\boldsymbol{\alpha} \cdot \mathbf{A}) \times \hat{\mathbf{p}}] \cdot \boldsymbol{\theta}
$$


The complete Hamiltonian $\hat{H}_{\mathrm{M}}^{\theta}$ (with all the terms up to the order $\left(m c^{2}\right)^{-2}$ ) are represented in the Appendix.

Taking as $\mathbf{A}$ a vector potential that corresponds to a homogeneous external magnetic field $\mathbf{B}=\left(B^{i}(t)\right)$ (in the symmetric gauge)

$$
A^{i}(x)=\frac{1}{2} \varepsilon_{i j k} B^{j}(t) x^{k},
$$

where $\varepsilon_{i j k}$ is the Levi-Civita symbol in three dimensions with the normalization $\varepsilon_{123}=1$, we obtain the following Hamiltonian:

$$
\begin{aligned}
& \hat{H}_{\mathrm{M}-\text { Nonrel }}^{\theta}=\frac{1}{2 m} \hat{\mathbf{P}}^{2}+e A_{0}-\frac{e}{2 \hbar}\left(\left[\nabla A_{0} \times \hat{\mathbf{p}}\right] \cdot \boldsymbol{\theta}\right) \\
& +\frac{e}{4 m \hbar c}(\hat{\mathbf{P}} \cdot[\mathbf{B} \times[\hat{\mathbf{p}} \times \boldsymbol{\theta}]])-\hat{\boldsymbol{\mu}} \cdot \mathbf{B}\left(1+\frac{e}{4 \hbar c}(\mathbf{B} \cdot \boldsymbol{\theta})\right),
\end{aligned}
$$

where $\hat{\boldsymbol{\mu}}$ is particle magnetic moment related to the spin operator $\hat{\mathbf{s}}$ as follows:

$$
\hat{\boldsymbol{\mu}}=\frac{e}{m c} \hat{\mathbf{s}}=\mu_{B} \boldsymbol{\sigma}, \quad \mu_{B}=\frac{e \hbar}{2 m c}, \quad \hat{\mathbf{s}}=\frac{\hbar}{2} \boldsymbol{\sigma}, \quad \boldsymbol{\sigma}=\left(\sigma^{i}\right) .
$$

The equation (13) (as well as eq. (11)) is not gauge invariant, since the Dirac equation (66) is not gauge invariant. That is why, we cannot interpret the factor in front of the operator $\hat{\boldsymbol{\mu}}$ as a physical magnetic field, this factor is gauge dependent. For example, choosing the Landau gauge $A^{1}=-B y, A^{2}=0$, this factor is reduced to $\mathbf{B}$ and does not depend on $\theta$ at all.

\section{$2.2 \theta$-modified pseudoclassical action for nonrelativistic spinning particle}

Considering the nonrelativistic limit of the equation (3), we obtain:

$$
\begin{aligned}
& i \hbar \partial_{t} \psi(x)=\hat{H}_{\mathrm{M}-\text { Nonrel }}^{\theta} \psi(x), \\
& \hat{H}_{\mathrm{M}-\text { Nonrel }}^{\theta}=\frac{1}{2 m} \hat{\mathbf{P}}^{2}(\hat{q})+e A^{0}(\hat{q})-\hat{\boldsymbol{\mu}} \cdot \mathbf{B}^{\theta}, \\
& \hat{\mathcal{B}}^{i}=-\frac{\varepsilon_{i j k}}{2} F_{j k}(\hat{q})=-\frac{\varepsilon_{i j k}}{2}\left\{F_{j k}(x)+\frac{i e}{\hbar c}\left[A_{j}(\hat{q}), A_{k}(\hat{q})\right]\right\},
\end{aligned}
$$

where

$$
\begin{aligned}
& F_{\mu \nu}(x)=\frac{i c}{\hbar e}\left[\hat{P}_{\mu}(x), \hat{P}_{\nu}(x)\right] \Longrightarrow \\
& F_{\mu \nu}(\hat{q})=\partial_{\mu} A_{\nu}(\hat{q})-\partial_{\nu} A_{\mu}(\hat{q})+\frac{i e}{\hbar c}\left[A_{\mu}(\hat{q}), A_{\nu}(\hat{q})\right] .
\end{aligned}
$$


If the external magnetic field is homogeneous, with potentials (12), it follows from (14) that $\mathbf{B}^{\theta}$ does not depend on spacial coordinates, is linear in $\theta$, and has the form 3 :

$$
\mathbf{B}^{\theta}=\left[1+\frac{e}{4 \hbar c}(\mathbf{B} \cdot \boldsymbol{\theta})\right] \mathbf{B} .
$$

In the case under consideration, one can construct a $\theta$-modified pseudoclassical action $S^{\theta}$ (à la Berezin-Marinov [36, 37]) for the nonrelativistic spinning particle. Such an action has the form

$$
\begin{aligned}
& S^{\theta}=\int d t L^{\theta}, \quad L^{\theta}=\mathbf{p} \cdot \dot{\mathbf{q}}-\frac{1}{2 m}\left(\mathbf{p}-\frac{e}{c} \mathbf{A}(\mathbf{q}, t)\right)^{2}-e A^{0}(\mathbf{q}) \\
& +i \boldsymbol{\xi} \cdot \dot{\boldsymbol{\xi}}+\frac{i e}{m c}\left(\mathbf{B}^{\theta} \cdot[\boldsymbol{\xi} \times \boldsymbol{\xi}]\right)-\frac{1}{2 \hbar} \dot{p}^{i} \theta^{i j} p^{j},
\end{aligned}
$$

where the variables $\mathbf{q}$ and $\mathbf{p}$ describe particle spacial movement, and Grassmann variables $\boldsymbol{\xi}=\left(\xi^{i}, i=1,2,3\right),\left[\xi^{i}, \xi^{j}\right]_{+}=0$, describe the particle spin. A quantization procedure presented below results in the nonrelativistic quantum mechanics of a spinning particle with the Hamiltonian $\hat{H}_{\mathrm{M}-\text { Nonrel }}^{\theta}$ defined by eq. (14) and commutation relations (4).

Treating (16) as a first order action in variables $\mathbf{q}, \mathbf{p}$, and $\boldsymbol{\xi}$, we introduce canonical momenta:

$$
\pi_{q i}=\frac{\partial L^{\theta}}{\partial \dot{q}^{i}}=p^{i}, \quad \pi_{p i}=\frac{\partial L^{\theta}}{\partial \dot{p}^{i}}=-\frac{1}{2 \hbar} \theta^{i j} p^{j}, \quad \pi_{\xi i}=\frac{\partial_{r} L^{\theta}}{\partial \dot{\xi}^{i}}=i \xi^{i} .
$$

Equations (17) imply the primary constraints $\Phi_{a}^{(1)}=\left(\Phi_{q i}^{(1)}, \Phi_{p i}^{(1)}, \Phi_{\xi i}^{(1)}\right)$,

$$
\Phi_{q i}^{(1)}=\pi_{q i}-p^{i}, \quad \Phi_{p i}^{(1)}=\pi_{p i}+\frac{1}{2 \hbar} \theta^{i j} p^{j}, \quad \Phi_{\xi i}^{(1)}=\pi_{\xi i}-i \xi^{i},
$$

which already are second-class constraints. Constructing the Hamiltonian $H^{(1)}=H^{\theta}+$ $\lambda^{a} \Phi_{a}^{(1)}$, according the canonical procedure [38], we obtain

$$
H^{\theta}=\frac{1}{2 m}\left(\mathbf{p}-\frac{e}{c} \mathbf{A}(\mathbf{q}, t)\right)^{2}+e A^{0}(\mathbf{q})-\frac{i e}{m c}\left(\mathbf{B}^{\theta} \cdot[\boldsymbol{\xi} \times \boldsymbol{\xi}]\right) .
$$

In the case under consideration all the Lagrange multipliers $\lambda^{a}$ can be fixed by the consistency conditions,

$$
\dot{\Phi}_{a}^{(1)}=0 \rightarrow \lambda^{a}=\left\{\Phi^{(1)}, \Phi^{(1)}\right\}_{a b}^{-1}\left\{\Phi_{b}^{(1)}, H\right\}
$$

Performing a canonical transformation to primed variables,

$$
q^{i \prime}=q^{i}, \quad p^{i}=p^{i}, \quad \pi_{q i}^{\prime}=\pi_{q i}-p^{i}, \quad \pi_{p i}^{\prime}=\pi_{p i}-q^{i}
$$

we obtain that the constraints in the new variables have the form:

$$
\Phi_{q i}^{\prime(1)}=\pi_{q i}^{\prime}=0, \quad \Phi_{p i}^{\prime(1)}=q^{i}+\pi_{p i}^{\prime}+\frac{1}{2 \hbar} \theta^{i j} p^{\prime j}=0 .
$$

\footnotetext{
${ }^{3}$ It should be noted that in the first order in $\theta$, the Hamiltonian (14) is reduced to the one (13).
} 
These constraints have a special form [38], such that variables $\left(q^{i \prime}, \pi_{q i}^{\prime}\right)$ can be eliminated from the consideration and for the rest of the variables $\left(p^{i}, \pi_{p i}^{\prime}\right)$, we have the total Hamiltonian

$$
\begin{aligned}
& H^{(1) \prime}=H_{*}^{\theta}+\lambda_{\xi}^{i} \Phi_{\xi i}^{(1)}, \quad \lambda_{\xi}^{i}=-\left\{\Phi_{\xi}^{(1)}, \Phi_{\xi}^{(1)}\right\}_{i j}^{-1}\left\{\Phi_{\xi j}^{(1)}, H\right\} \\
& H_{*}^{\theta}=H^{\theta}\left(-\pi_{p i}^{\prime}-\frac{1}{2 \hbar}\left(\theta^{i j} p^{\prime j}\right), p^{\prime} ; \xi\right) .
\end{aligned}
$$

After an additional canonical transformation $p^{i}=p^{i}, \quad \pi_{p i}^{\prime}=-x^{i}$, i.e., $q^{i}=x^{i}-\frac{1}{2 \hbar} \theta^{i k} p^{k}$ , the Hamiltonian $H_{*}^{\theta}$ takes the form $H^{\theta}=H^{\theta}(q, p ; \xi)$.

Now, we can pass to Dirac brackets and the Hamiltonian $H^{\theta}(q, p ; \xi)$. The only nonzero Dirac brackets between the remaining variables are:

$$
\begin{aligned}
& \left\{x^{i}, p^{j}\right\}_{D(\Phi)}=\delta^{i j}, \quad\left\{\xi^{i}, \xi^{j}\right\}_{D(\Phi)}=-\frac{i}{2} \delta^{i j}, \\
& \left\{q^{i}, q^{j}\right\}_{D(\Phi)}=\frac{1}{\hbar} \theta^{i j}, \quad\left\{q^{i}, p^{j}\right\}_{D(\Phi)}=\delta^{i j} .
\end{aligned}
$$

Proceeding to the quantization, we assign operators to classical variables $(q, p, \xi)$, such that the only nonzero commutators are

$$
\begin{aligned}
& {\left[\hat{q}^{i}, \hat{q}^{j}\right]=\left.i \hbar\left\{q^{i}, q^{j}\right\}_{D(\Phi)}\right|_{\eta=\hat{\eta}}=i \theta^{i j},} \\
& {\left[\hat{x}^{i}, \hat{p}^{j}\right]=\left[\hat{q}^{i}, \hat{p}^{j}\right]=\left.i \hbar\left\{q^{i}, p^{j}\right\}_{D(\Phi)}\right|_{\eta=\hat{\eta}}=i \hbar \delta^{i j},} \\
& {\left[\hat{\xi}^{i}, \hat{\xi}^{j}\right]_{+}=\left.i \hbar\left\{\xi^{i}, \xi^{j}\right\}_{D(\Phi)}\right|_{\eta=\hat{\eta}}=\frac{\hbar}{2} \delta^{i j} .}
\end{aligned}
$$

The algebra (22) can be realized in a Hilbert space, whose elements $\psi(x)$ are two components spinors dependent on $x$, such that,

$$
\hat{x}^{i}=x^{i}, \quad \hat{p}^{i}=-i \hbar \frac{\partial}{\partial x^{i}}, \quad \hat{q}^{i}=x^{i}+\frac{i}{2} \theta^{i j} \partial_{j}, \quad \hat{\xi}^{i}=\frac{\sqrt{\hbar}}{2} \sigma^{i} .
$$

It follows from (23) and (21) that the corresponding quantum Hamiltonian coincides with the Hamiltonia (14), and the nonrelativistic spin operator reads $\hat{\mathbf{s}}=-i[\hat{\boldsymbol{\xi}} \times \hat{\boldsymbol{\xi}}]=(\hbar / 2) \boldsymbol{\sigma}$.

\subsection{Consideration in the framework of SW map}

Here we are going to obtain a $\theta$-modified Dirac equation from the SW map applied to the action (11). It is known that such a modified action is already gauge invariant under the gauge transformations

$$
\check{U}_{\check{\lambda}}(x)=\left(e^{i \check{\lambda}(x)}\right)_{\star}=1+i \check{\lambda}(x)-(1 / 2) \check{\lambda}(x) \star \check{\lambda}(x)+O\left(\check{\lambda}^{3}\right),
$$


where $\check{\lambda}(x)$ is the noncommutative gauge parameter, [21]. At the first step, we take the action

$$
\begin{aligned}
& S_{\mathrm{SW}}^{\theta}=\int d^{4} x \mathcal{L}_{\mathrm{SW}}^{\theta}, \quad \mathcal{L}_{\mathrm{SW}}^{\theta}=\bar{\Psi}(x) \star\left(\gamma^{\mu} \check{P}_{\mu}-m c\right) \star \check{\Psi}(x), \\
& \check{F}^{\mu \nu}(x)=\partial^{\mu} \check{A}^{\nu}(x)-\partial^{\nu} \check{A}^{\mu}(x)+\frac{i e}{\hbar c}\left[\check{A}^{\mu}(x) \star \check{A}^{\nu}(x)\right], \\
& {\left[\check{A}^{\mu}(x) \star \check{A}^{\nu}(x)\right]=\check{A}^{\mu}(x) \star \check{A}^{\nu}(x)-\check{A}^{\nu}(x) \star \check{A}^{\mu}(x),} \\
& {\left[\check{P}_{\mu}^{\star} \check{P}_{\nu}\right]=-\frac{i \hbar e}{c} \check{F}^{\mu \nu}(x),}
\end{aligned}
$$

where (in the first order in $\theta$ ) the SW fields $\check{A}_{\mu}(x)$ and $\check{\Psi}(x)$ are expressed via the ordinary field $A_{\mu}(x)$ and $\Psi(x)$ as:

$$
\begin{aligned}
& \check{A}_{\mu}(x)=A_{\mu}(x)+\frac{e}{2 \hbar c} \theta^{\alpha \beta} A_{\alpha}(x)\left(\partial_{\beta} A_{\mu}(x)+F_{\beta \mu}(x)\right)+O\left(\theta^{2}\right), \\
& \check{\Psi}(x)=\Psi(x)+\frac{e}{2 \hbar c} \theta^{\alpha \beta} A_{\alpha}(x) \partial_{\beta} \Psi(x)+O\left(\theta^{2}\right) .
\end{aligned}
$$

set4 [21, 22, 23, 24, 25, 26]. Then we consider the star product in the first order in $\theta$ and finally we obtain the spinor field action (e.g. [25, 26]),

$$
\mathcal{L}_{\mathrm{SW}}^{\theta}=\bar{\Psi}(x)\left\{\gamma^{\mu}\left[\left(1+\frac{e}{4 \hbar c} \theta^{\alpha \beta} F_{\alpha \beta}\right) \hat{P}_{\mu}-\frac{e}{2 \hbar c} \theta^{\alpha \beta} F_{\alpha \mu} \hat{P}_{\beta}\right]-m c\left(1+\frac{e}{4 \hbar c} \theta^{\alpha \beta} F_{\alpha \beta}\right)\right\} \Psi(x) .
$$

We identify the Euler-Lagrange equation $\delta S_{\mathrm{SW}}^{\theta} / \delta \bar{\Psi}(x)=0$ with the $\theta$-mod. Dirac equation from SW map. For $\theta^{0 \mu}=0$, we obtain:

$$
\begin{aligned}
& i \hbar \partial_{t} \Psi=\left(\hat{\mathbb{H}}_{\mathrm{D}}+\Delta \hat{\mathbb{H}}_{\mathrm{SW}}^{\theta}\right) \Psi, \\
& \Delta \hat{\mathbb{H}}_{\mathrm{SW}}^{\theta}=\frac{e}{2 \hbar}\{([\mathbf{E} \times \hat{\mathbf{P}}] \cdot \boldsymbol{\theta})+([\boldsymbol{\theta} \times[\boldsymbol{\alpha} \times \mathbf{B}]] \cdot \hat{\mathbf{P}})\}, \\
& \mathbf{E}=\left(E^{i}=F^{i 0}\right), \quad \mathbf{B}=\boldsymbol{\nabla} \times \mathbf{A} .
\end{aligned}
$$

We stress that this equation is already gauge invariant under $U_{\lambda}(1)$ gauge transformations.

In the same manner, as it was done in the previous subsection, we can derive the nonrelativistic limit of the obtained equation. As a result, we obtain the following Schrödinger equation for a spinor $\psi(x)$ :

$$
\begin{aligned}
& i \hbar \partial_{t} \psi(x)=\hat{H}_{\mathrm{SW}}^{\theta} \psi(x), \\
& \hat{H}_{\mathrm{SW}}^{\theta}=\frac{1}{2 m c^{2}} \mathcal{O}_{\mathrm{SW}}^{2}+e A_{0}+\frac{e}{2 \hbar}([\mathbf{E} \times \hat{\mathbf{P}}] \cdot \boldsymbol{\theta}), \\
& \mathcal{O}_{\mathrm{SW}}=c\left\{\boldsymbol{\alpha}+\frac{e}{2 \hbar c}[\boldsymbol{\theta} \times[\boldsymbol{\alpha} \times \mathbf{B}]]\right\} \cdot \hat{\mathbf{P}} .
\end{aligned}
$$

\footnotetext{
${ }^{4}$ Here the noncommutative SW fields are labeled by a "check" above.
} 
Complete operators $\mathcal{O}_{\mathrm{SW}}^{2}$ and $\hat{H}_{\mathrm{SW}}^{\theta}$ (with all the terms up to the order $\left(m c^{2}\right)^{-2}$ ) are presented in the Appendix.

If we restrict ourselves by external homogeneous magnetic field $\mathbf{B}=\left(B^{i}(t)\right)$ only, we reduce the Hamiltonian $\hat{H}_{\mathrm{SW}}^{\theta}$ to the following form:

$$
\begin{aligned}
& \hat{H}_{\mathrm{SW}-\text { Pauli }}^{\theta}=\frac{1}{2 m} \hat{\mathbf{P}}^{2}+e A_{0}+\frac{e}{2 \hbar}([\mathbf{E} \times \hat{\mathbf{P}}] \cdot \boldsymbol{\theta}) \\
& +\frac{e}{2 m \hbar c}(\hat{\mathbf{P}} \cdot[\mathbf{B} \times[\hat{\mathbf{P}} \times \boldsymbol{\theta}]])-\hat{\boldsymbol{\mu}} \cdot \mathbf{B}_{\mathrm{SW}}^{\theta},
\end{aligned}
$$

where

$$
\mathbf{B}_{\mathrm{SW}}^{\theta}=\left[1+\frac{e}{\hbar c}(\mathbf{B} \cdot \boldsymbol{\theta})\right] \mathbf{B} .
$$

In contrast to the nonrelativistic equation with the Hamiltonian (13), equation (27), from our point of view, is a good candidate to be considered the $\theta$-modified Pauli equation, since $\hat{H}_{\mathrm{SW}-\text { Pauli }}^{\theta}$ is gauge invariant. For the same reason, we can identify now the quantity $\mathbf{B}_{\mathrm{SW}}^{\theta}$ with a physical ( $\theta$-modified) magnetic field.

It should be noted that there exists an original manner to obtain a nonrelativistic wave equation for spinning particles from group theoretical considerations presented in [39]. It should be interesting to see if similar considerations (maybe suitable modified due to the well-known problems with the classical space-time symmetries in noncommutative spaces, e.g. with the Lorentz invariance in the relativistic case, see e.g. [40]) can work in the noncommutative case.

\section{Two spins in a noncommutative space}

It is known, that a reduction of the Pauli equation to the $(0+1)$-dimensional case, allows one to obtain the so-called spin equation (in absence of the scalar potential, $A_{0}=0$ ), which describes a motion of a spatially "frozen" spin in a magnetic field, see [35]. The same reduction of the equation (28) yields the spin equation in the noncommutative space, or $\theta$-modified spin equation:

$$
i \hbar \partial_{t} \psi=-\hat{\boldsymbol{\mu}} \cdot \mathbf{B}\left(1+\frac{e}{\hbar c}(\mathbf{B} \cdot \boldsymbol{\theta})\right) \psi
$$

In the commutative space, the spin equation for two interaction spins in a homogeneous magnetic field can be written as [41,

$$
\begin{aligned}
& i \hbar \partial_{t} \Psi(t)=\hat{H}\left(\mathbf{B}_{1}, \mathbf{B}_{2}, J\right) \Psi(t), \hat{H}\left(\mathbf{B}_{1}, \mathbf{B}_{2}, J\right)=\boldsymbol{\rho} \cdot \mathbf{B}_{1}+\boldsymbol{\Sigma} \cdot \mathbf{B}_{2}+\frac{J}{2} \boldsymbol{\Sigma} \cdot \boldsymbol{\rho}, \\
& \boldsymbol{\Sigma}=I \otimes \boldsymbol{\sigma}, \boldsymbol{\rho}=\boldsymbol{\sigma} \otimes I, \quad(\boldsymbol{\Sigma} \cdot \boldsymbol{\rho})=\boldsymbol{\sigma} \otimes \boldsymbol{\sigma}=\sum_{i=1}^{3} \sigma_{i} \otimes \sigma_{i},
\end{aligned}
$$


where $I$ stand for a $2 \times 2$ unit matrix. The first (second) term on $\hat{H}$ represents the Pauli interaction of the first (second) spin with the magnetic field $\mathbf{B}_{1}(t)\left(\mathbf{B}_{2}(t)\right)$ and the last term a spherically symmetric interaction $J=J(t)$ between the two spins (a Heisenberg interaction). In the above expression we are absorbing the magnetic momentum of the particle on the magnetic field $\left(-\mu_{B} \mathbf{B} \equiv \mathbf{B}\right)$. In this manner a different effective field in each spin can be obtained by using particles with different magnetic moments.

As we know from the result (28), the noncommutative Pauli interaction for a spin in a homogeneous magnetic field can be obtained by the replacement $\mathbf{B}_{a}(t) \rightarrow \mathbf{B}_{a}^{\theta}(t), a=1,2$, see eq. (29). So, if the fields $\mathbf{B}_{a}$ are parallel (we choose them in the $z$-direction) in the noncommutative case the Hamiltonian (31) becomes

$$
\begin{aligned}
& \hat{H}^{\theta}=\frac{1}{2}\left[\left(\Sigma_{3}+\rho_{3}\right) B_{+}^{\theta}-\left(\Sigma_{3}-\rho_{3}\right) B_{-}^{\theta}-J^{\theta}\right]+A J^{\theta}, \\
& B_{ \pm}^{\theta}(t)=B_{1}^{\theta}(t) \pm B_{2}^{\theta}(t), \quad A=\frac{1}{2}[1+(\boldsymbol{\Sigma} \cdot \boldsymbol{\rho})],
\end{aligned}
$$

where we are supposing that the new noncommutative interaction $J^{\theta}$ remains spherically symmetric. Using the techniques described in [42], it is possible to show that the evolution operator for the Schrödinger equation with the above Hamiltonian has the form

$$
U(t)=\left(\begin{array}{ccc}
f_{+}(t) & 0 & 0 \\
0 & \hat{u}(t) & 0 \\
0 & 0 & f_{-}(t)
\end{array}\right), \quad f_{ \pm}(t)=\exp \left[-i \int_{0}^{t}\left(\frac{J^{\theta}}{2} \pm B_{+}\right) d \tau\right]
$$

where the $2 \times 2$ matrix $\hat{u}$ is the evolution operator for the following two level system problem [35],

$$
i \partial_{t} \psi=\left[(\boldsymbol{\sigma} \cdot \mathbf{K})-J^{\theta} / 2\right] \psi, \quad \mathbf{K}(t)=\left(J^{\theta}(t), 0, B_{-}^{\theta}(t)\right) .
$$

Having this operator in the explicit form, one can calculate the probability transition $P(t)$ between any states. An interesting special case is the transition between the two orthogonal EPR states $\left|\Psi_{ \pm}\right\rangle$,

$$
\begin{aligned}
& \left|\Psi_{ \pm}\right\rangle=\frac{1}{\sqrt{2}}[|++\rangle \pm|--\rangle],| \pm \pm\rangle=| \pm\rangle \otimes| \pm\rangle, \\
& |+\rangle=\left(\begin{array}{l}
1 \\
0
\end{array}\right), \quad|-\rangle=\left(\begin{array}{l}
0 \\
1
\end{array}\right),
\end{aligned}
$$

once, in this case, this probability does not depends on $\hat{u}$ neither on the unknown function $J^{\theta}$

$$
P(t)=\left|\left\langle\Psi_{+}|U(t)| \Psi_{-}\right\rangle\right|^{2}=\left|\sin \left[2 \int_{0}^{t} B_{+}^{\theta} d \tau\right]\right|^{2} .
$$


In particular, for the two dephased fields

$$
B_{1}=B \cos (\omega t), \quad B_{2}=B \cos (\omega t+\phi), \quad \phi=\pi(1+2 n), \quad n \in \mathbb{N},
$$

where $B$ and $\omega$ are constants, we have a strictly dependent $\theta$ transition, which is zero unless $\theta$ is nonzerd 5 ,

$$
P(t)=\left|\sin \left[\theta \frac{2 e \mu_{B} B^{2}}{\hbar^{2} c}\left(t+\frac{\sin 2 \omega t}{2 \omega}\right)\right]\right|^{2} .
$$

This result can be used to obtain an upper bound on $\theta$. For such an estimation, we suppose that the magnetic field is strong enough but realistic for laboratory conditions, let say $B=10 \mathrm{~T}$, the transition time is $t=1 \mathrm{~s}$, and the resolution of the experiment allows us to measure the probability with the precision $0,05 \%$, i.e., $P(t)<0.005$. Then with these numbers, we obtain the following upper bound on $\theta$ :

$$
P(t)<0.005 \rightarrow|\theta| \lesssim 2.65 \times 10^{-30} \mathrm{~m}^{2}
$$

This result matches with another estimation, which could be obtained from energy splitting of the hydrogen atom due to the space noncommutativity [9].

\section{Summary}

Starting with the two $\theta$-modified spinor field actions, the first one obtained by a simple Moyal modification and the second one by the SW map, we derive and discuss two different $\theta$-modified Dirac equations. Both actions were already known before, see references above, however, the $\theta$-modified Dirac equation from the SW map was represented for the first time.

Considering the nonrelativistic limit in both Dirac equations, we derived two Schrödinger equations for nonrelativistic spinning particles in the noncommutative space. One of these equations is gauge invariant with respect to $U(1)$ gauge transformations of the external electromagnetic field and is interpreted by us as a $\theta$-modified Pauli equation.

Such an equation allows us to extract a $\theta$-modified nonrelativistic interaction of the magnetic field with the particle magnetic moment. Using the latter result, we construct a $\theta$ modified spin equation, which describes a $\theta$-modified two-level system, and then a $\theta$-modified Heisenberg model for two coupled spins placed in an external magnetic field.

A pseudoclassical model for a nonrelativistic spinning particle in the noncommutative space is constructed. Its quantization leads to one of the $\theta$-modified wave equation for such a particle.

\footnotetext{
${ }^{5}$ Here, we have restored magnetic momentum $\mu_{B}$.
} 
In the framework of the Heisenberg model, we calculate the probability transition between two orthogonal EPR (Einstein-Podolsky-Rosen) states for a pair of spins in an oscillatory magnetic field and show that some of such transitions, which are forbidden in the commutative space, are possible due to the space noncommutativity. This allows us to estimate an upper bound on the noncommtativity parameter.

\section{Acknowledgements}

We are indebted to Prof. Peter A. Horvathy for the helpful commentaries about the references. T.C.A. thanks FAPESP; M.C.B. thanks FAPESP and D.M.G. thanks FAPESP and CNPq for permanent support.

\section{Appendix}

The complete nonrelativistic Hamiltonian $\hat{H}_{\mathrm{M}}^{\theta}$ (11), with terms of the order $\left(m c^{2}\right)^{-2}$ is

$$
\begin{aligned}
& \hat{H}_{\mathrm{M}}^{\theta}=\frac{1}{2 m c^{2}} \mathcal{O}_{\mathrm{M}}^{2}+e A_{0}-\frac{e}{2 \hbar}\left[\nabla A_{0} \times \hat{\mathbf{p}}\right] \cdot \boldsymbol{\theta} \\
& -\frac{1}{8 m^{2} c^{4}}\left[\mathcal{O}_{\mathrm{M}},\left(e\left[\mathcal{O}_{\mathrm{M}}, A_{0}\right]+i \hbar \partial_{t} \mathcal{O}_{\mathrm{M}}-\frac{e}{2 \hbar} \varepsilon_{i j k}\left[\mathcal{O}_{\mathrm{M}}, \partial_{i} A_{0} \hat{p}^{j}\right] \theta^{k}\right)\right],
\end{aligned}
$$


where the operators above are

$$
\begin{aligned}
\mathcal{O}_{\mathrm{M}}^{2} & =c^{2} \hat{\mathbf{P}}^{2}-e \hbar c(\mathbf{B} \cdot \boldsymbol{\sigma})-\frac{e c}{\hbar}\left\{\left(\left[\hat{\mathbf{p}} \times \boldsymbol{\nabla} A^{i}\right] \cdot \boldsymbol{\theta}\right) \hat{P}^{i}-\frac{i \hbar}{2}[\hat{\mathbf{p}} \times \boldsymbol{\nabla}(\nabla \cdot \mathbf{A})] \cdot \boldsymbol{\theta}\right. \\
& \left.-\frac{\hbar}{2}[\boldsymbol{\nabla}([\boldsymbol{\nabla} \times \mathbf{A}] \cdot \boldsymbol{\sigma}) \times \hat{\mathbf{p}}] \cdot \boldsymbol{\theta}+\frac{e \hbar}{2 c}\left[\varepsilon_{i j k}\left(\boldsymbol{\nabla} A^{i}\right) \times\left(\boldsymbol{\nabla} A^{j}\right) \sigma^{k}\right] \cdot \boldsymbol{\theta}\right\}, \\
{\left[\mathcal{O}_{\mathrm{M}},\left[\mathcal{O}_{\mathrm{M}}, A_{0}\right]\right] } & =-\hbar c^{2}\left\{\hbar \boldsymbol{\nabla}^{2} A_{0}+2\left[\left(\boldsymbol{\nabla} A_{0}\right) \times \hat{\mathbf{P}}\right] \cdot \boldsymbol{\sigma}\right\} \\
& -\frac{e \hbar c}{2}\left\{\partial_{i}\left[\left(\boldsymbol{\nabla} A^{i}\right) \times\left(\boldsymbol{\nabla} A_{0}\right)\right] \cdot \boldsymbol{\theta}+\left[\left(\boldsymbol{\nabla} A^{i}\right) \times \partial_{i}\left(\boldsymbol{\nabla} A_{0}\right)\right] \cdot \boldsymbol{\theta}\right. \\
& \left.+i \varepsilon_{i j k}\left(\partial_{i}\left[\left(\boldsymbol{\nabla} A^{j}\right) \times\left(\boldsymbol{\nabla} A_{0}\right)\right] \cdot \boldsymbol{\theta}\right) \sigma^{k}+i \varepsilon_{i j k}\left(\left[\left(\boldsymbol{\nabla} A^{i}\right) \times \partial_{j}\left(\boldsymbol{\nabla} A_{0}\right)\right] \cdot \boldsymbol{\theta}\right) \sigma^{k}\right\} \\
& -e c\left\{\varepsilon_{i j k}\left(\left[\left(\boldsymbol{\nabla} A^{i}\right) \times\left(\boldsymbol{\nabla} A_{0}\right)\right] \cdot \boldsymbol{\theta}\right) \hat{P}^{j} \sigma^{k}-\varepsilon_{i j k}\left(\left[\left(\partial_{i} \mathbf{A}\right) \times\left(\boldsymbol{\nabla} A_{0}\right)\right] \cdot \boldsymbol{\sigma}\right) \hat{p}^{j} \theta^{k}\right\} \\
{\left[\mathcal{O}_{\mathrm{M}}, \partial_{t} \mathcal{O}_{\mathrm{M}}\right] } & =e \hbar c\left\{i \partial_{t}(\boldsymbol{\nabla} \cdot \mathbf{A})-\left[\boldsymbol{\nabla} \times\left(\partial_{t} \mathbf{A}\right)\right] \cdot \boldsymbol{\sigma}+\frac{2 i}{\hbar}\left[\left(\partial_{t} \mathbf{A}\right) \times \hat{\mathbf{P}}\right] \cdot \boldsymbol{\sigma}\right\} \\
& -\frac{e c}{2}\left\{i\left[\boldsymbol{\nabla}\left(\boldsymbol{\nabla} \cdot \partial_{t} \mathbf{A}\right) \times \hat{\mathbf{p}}\right] \cdot \boldsymbol{\theta}-\left[\boldsymbol{\nabla}\left(\left[\boldsymbol{\nabla} \times\left(\partial_{t} \mathbf{A}\right)\right] \cdot \boldsymbol{\sigma}\right) \times \hat{\mathbf{p}}\right] \cdot \boldsymbol{\theta}\right. \\
& \left.+\frac{i}{\hbar} \varepsilon_{i j k}\left(\left[\partial_{t}\left(\boldsymbol{\nabla} A^{i}\right) \times \hat{\mathbf{p}}\right] \cdot \boldsymbol{\theta}\right) \hat{P}^{j} \sigma^{k}+2 i \frac{e}{c}\left[\nabla\left(\partial_{t} A^{i}\right) \times\left(\boldsymbol{\nabla} A^{i}\right)\right] \cdot \boldsymbol{\theta}\right\} \\
& +e^{2} \varepsilon_{i j k}\left\{\left(\left[\boldsymbol{\nabla}\left(\partial_{t} A^{i}\right) \times\left(\boldsymbol{\nabla} A^{j}\right)\right] \cdot \boldsymbol{\theta}\right) \sigma^{k}-\frac{i}{\hbar}\left(\left[\left(\partial_{i} \mathbf{A}\right) \times\left(\partial_{t} \mathbf{A}\right)\right] \cdot \boldsymbol{\sigma}\right) \hat{p}^{j} \theta^{k}\right\} \\
{\left[\mathcal{O}_{\mathrm{M}},\left[\mathcal{O}_{\mathrm{M}}, E^{i} \hat{p}^{j}\right]\right] } & =-\hbar^{2} c^{2}\left\{\left(\boldsymbol{\nabla}^{2} E^{i}\right) \hat{p}^{j}+\frac{e}{c}\left(\partial_{l} E^{i}\right)\left(\partial_{j} A^{l}\right)+\frac{e}{c} \partial_{l}\left(E^{i} \partial_{j} A^{l}\right)\right\} \\
& +i e \hbar c\left\{\hbar\left[\left(\boldsymbol{\nabla} E^{i}\right) \times\left(\partial_{j} \mathbf{A}\right)\right] \cdot \boldsymbol{\sigma}-\hbar\left[\boldsymbol{\nabla} \times\left(E^{i} \partial_{j} \mathbf{A}\right)\right] \cdot \boldsymbol{\sigma}\right. \\
& \left.-2 i \hbar^{2}\left[\left(\nabla E^{i}\right) \times\left(\partial_{j} \mathbf{A}\right)\right] \cdot \boldsymbol{\sigma}+2 \hbar c\left(\left[\left(\nabla E^{i}\right) \times \hat{\mathbf{P}}\right] \cdot \boldsymbol{\sigma}\right) \hat{p}^{j}\right\} .
\end{aligned}
$$

The complete nonrelativistic Hamiltonian $\hat{H}_{\mathrm{SW}}^{\theta}$ (27), with terms of the order $\left(m c^{2}\right)^{-2}$ reads

$$
\begin{aligned}
& \hat{H}_{\mathrm{SW}}^{\theta}=m c^{2} \gamma^{0}+e A_{0}+\frac{e}{2 \hbar}[\mathbf{E} \times \hat{\mathbf{P}}] \cdot \boldsymbol{\theta}+\frac{1}{2 m c^{2}} \gamma^{0} \mathcal{O}_{\mathrm{SW}}^{2} \\
& -\frac{1}{8 m^{2} c^{4}}\left[\mathcal{O}_{\mathrm{SW}},\left(e\left[\mathcal{O}_{\mathrm{SW}}, A_{0}\right]+i \hbar \partial_{t} \mathcal{O}_{\mathrm{SW}}+\frac{e}{2 \hbar} \varepsilon_{i j k}\left[\mathcal{O}_{\mathrm{SW}}, E^{i} \hat{P}^{j}\right]\right) \theta^{k}\right]
\end{aligned}
$$

where 


$$
\begin{aligned}
& \mathcal{O}_{\mathrm{SW}}^{2}=\left(c^{2}+\frac{e c}{\hbar}(\boldsymbol{\theta} \cdot \mathbf{B})\right)\left(\hat{\mathbf{P}}^{2}-\frac{\hbar e}{c} \mathbf{B} \cdot \boldsymbol{\sigma}\right)-\frac{e c}{\hbar}(\mathbf{B} \cdot \hat{\mathbf{P}})(\boldsymbol{\theta} \cdot \hat{\mathbf{P}}) \\
& -i \frac{e c}{2}\left\{\boldsymbol{\nabla}(\boldsymbol{\theta} \cdot \mathbf{B}) \cdot \hat{\mathbf{P}}-\left(\boldsymbol{\theta} \cdot \boldsymbol{\nabla} B^{i}\right) \hat{P}^{i}+i[\boldsymbol{\nabla}(\boldsymbol{\theta} \cdot \mathbf{B}) \times \hat{\mathbf{P}}] \cdot \boldsymbol{\sigma}\right. \\
& \left.-i\left(\left[\left(\boldsymbol{\nabla} B^{i}\right) \times \boldsymbol{\theta}\right] \cdot \boldsymbol{\sigma}\right) \hat{P}^{i}\right\} \text {, } \\
& {\left[\mathcal{O}_{\mathrm{SW}},\left[\mathcal{O}_{\mathrm{SW}}, A_{0}\right]\right]=-\hbar c^{2}\left(1+\frac{e}{\hbar c} \boldsymbol{\theta} \cdot \mathbf{B}\right)\left\{\hbar \boldsymbol{\nabla}^{2} A_{0}+2\left[\boldsymbol{\nabla} A_{0} \times \hat{\mathbf{P}}\right] \cdot \boldsymbol{\sigma}\right\}} \\
& +e c \hbar\left\{(\mathbf{B} \cdot \boldsymbol{\nabla})\left(\boldsymbol{\theta} \cdot \boldsymbol{\nabla} A_{0}\right)-\frac{1}{2}\left(\boldsymbol{\nabla} A_{0} \cdot \boldsymbol{\nabla}(\boldsymbol{\theta} \cdot \mathbf{B})\right)\right. \\
& +\frac{1}{2}(\boldsymbol{\theta} \cdot \boldsymbol{\nabla} \mathbf{B}) \cdot\left(\boldsymbol{\nabla} A_{0}\right)-\frac{1}{\hbar}\left(\mathbf{B} \cdot \boldsymbol{\nabla} A_{0}\right)[\hat{\mathbf{P}} \times \boldsymbol{\theta}] \cdot \boldsymbol{\sigma} \\
& \left.+\frac{1}{\hbar}\left(\left[\boldsymbol{\nabla} A_{0} \times \boldsymbol{\theta}\right] \cdot \boldsymbol{\sigma}\right)(\mathbf{B} \cdot \hat{\mathbf{P}})\right\} \text {, } \\
& {\left[\mathcal{O}_{\mathrm{SW}}, \partial_{t} \mathcal{O}_{\mathrm{SW}}\right]=e c\left(1+\frac{e}{\hbar c}(\boldsymbol{\theta} \cdot \mathbf{B})\right)\left\{i \hbar \partial_{t}(\boldsymbol{\nabla} \cdot \boldsymbol{A})+2 i\left[\left(\partial_{t} \mathbf{A}\right) \times \hat{\mathbf{P}}\right] \cdot \boldsymbol{\sigma}-\hbar\left(\partial_{t} \mathbf{B} \cdot \boldsymbol{\sigma}\right)\right\}} \\
& -\frac{i e^{2}}{2 \hbar}\left\{\hbar \mathbf{B} \cdot \boldsymbol{\nabla}\left(\left(\partial_{t} \mathbf{A} \cdot \boldsymbol{\theta}\right)+\left(\left[\partial_{t} \mathbf{A} \times \boldsymbol{\theta}\right]\right) \cdot \boldsymbol{\sigma}\right)+2\left(\left[\partial_{t} \mathbf{A} \times \boldsymbol{\theta}\right] \cdot \boldsymbol{\sigma}\right)(\mathbf{B} \cdot \hat{\mathbf{P}})\right\} \\
& +i \frac{e c}{2}\left\{-\boldsymbol{\nabla}\left(\boldsymbol{\theta} \cdot \partial_{t} \mathbf{B}\right) \cdot \hat{\mathbf{P}}+\left(\boldsymbol{\theta} \cdot \boldsymbol{\nabla} \partial_{t} B^{i}\right) \hat{P}^{i}+i\left[\boldsymbol{\nabla}\left(\partial_{t} \mathbf{B} \cdot \boldsymbol{\theta}\right) \times \hat{\mathbf{P}}\right] \cdot \boldsymbol{\sigma}\right. \\
& \left.-i\left(\left[\nabla \partial_{t} B^{i} \times \boldsymbol{\theta}\right] \cdot \boldsymbol{\sigma}\right) \hat{P}^{i}-\frac{2}{\hbar}\left(\left(\partial_{t} \mathbf{B}\right) \cdot \hat{\mathbf{P}}\right)[\hat{\mathbf{P}} \times \boldsymbol{\theta}] \cdot \boldsymbol{\sigma}\right\} \\
& +\frac{e^{2}}{2}\left\{i\left(\partial_{t} \mathbf{A}\right) \cdot \boldsymbol{\nabla}(\boldsymbol{\theta} \cdot \mathbf{B})-i \boldsymbol{\theta} \cdot \boldsymbol{\nabla}\left(\mathbf{B} \cdot \partial_{t} \mathbf{A}\right)-\left(\boldsymbol{\theta} \cdot \partial_{t} \mathbf{B}\right)(\mathbf{B} \cdot \boldsymbol{\sigma})\right. \\
& +\left[\boldsymbol{\nabla}\left(\mathbf{B} \cdot \partial_{t} \mathbf{A}\right) \times \boldsymbol{\theta}\right] \cdot \boldsymbol{\sigma}-\left[\boldsymbol{\nabla} \times\left(\partial_{t} \mathbf{A}(\mathbf{B} \cdot \boldsymbol{\theta})\right)\right] \cdot \boldsymbol{\sigma}+2\left(\partial_{t} \mathbf{B} \cdot \boldsymbol{\theta}\right)(\mathbf{B} \cdot \boldsymbol{\sigma}) \\
& \left.+i\left[\left(\partial_{t} \mathbf{B}\right) \times \boldsymbol{\theta}\right] \cdot \mathbf{B}+\frac{2 i}{\hbar}\left(\mathbf{B} \cdot \partial_{t} \mathbf{A}\right)[\hat{\mathbf{P}} \times \boldsymbol{\theta}] \cdot \boldsymbol{\sigma}\right\}, \\
& {\left[\mathcal{O}_{\mathrm{SW}},\left[\mathcal{O}_{\mathrm{SW}}, E^{i} \hat{P}^{j}\right]\right]=-\hbar^{2} c^{2}\left\{\left(\nabla^{2} E^{i}\right) \hat{P}^{j}-\frac{2 e}{c} \varepsilon_{j k l} B^{k}\left(\partial_{l} E^{i}\right)+\frac{e}{c} \varepsilon_{j l k} E^{i} \partial_{l} B^{k}\right.} \\
& -\frac{2}{\hbar}\left(\left[\left(\nabla E^{i}\right) \times \hat{\mathbf{P}}\right] \cdot \boldsymbol{\sigma}\right) \hat{P}^{j}+\frac{i e}{\hbar c} E^{i} \partial_{j}(\mathbf{B} \cdot \boldsymbol{\sigma}) \\
& -i \frac{e}{c} E^{i} \sigma^{j}(\nabla \cdot \mathbf{B})-\frac{2 e}{\hbar c}(\mathbf{B} \cdot \boldsymbol{\sigma}) E^{i} \hat{P}^{j}+\frac{2 e}{\hbar c} E^{i} \sigma^{j}(\mathbf{B} \cdot \hat{\mathbf{P}}) \\
& \left.+\frac{2 i e}{c}\left(\partial_{j} E^{i}\right)(\mathbf{B} \cdot \boldsymbol{\sigma})-\frac{2 i e}{c}\left(\mathbf{B} \cdot \boldsymbol{\nabla} E^{i}\right) \sigma^{j}\right\} \text {. }
\end{aligned}
$$

\section{References}

[1] S. Bourouaine and A. Benslama, J. Phys. A: Math. Gen. 35, 7389 (2005).

[2] D. M. Gitman and V. G. Kupriyanov, Eur. Phys. J. C 54, 325 (2008). 
[3] B. Chakraborty, S. Gangopadhyay and A. Saha, Phys. Rev. D 70, 107707 (2004).

[4] E. Harikumar, V. Sunil Kumar and A. Khare, Phys. Lett. B 589, 155 (2004).

[5] A. Kokado, T. Okamura and T. Saito, Phys. Rev. D 69, 125007 (2004).

[6] F. G. Scholtz, B. Chakraborty, S. Gangopadhyay and A. G. Hazra, Phys. Rev. D 71, 085005 (2005).

[7] A. Smailagic and E. Spallucci, Phys. Rev. D 65, 107701 (2002); J. Phys. A: Math. Gen. 35, L363 (2002).

[8] F. Delduc, Q. Duret, F. Gieres and M. Lefrançois, J. Phys. Conf. Ser. 103, 012020 (2008).

[9] T. C. Adorno, M. C. Baldiotti, M. Chaichian, D. M. Gitman and A. Tureanu, Phys. Lett. B 682, 235 (2009).

[10] I. Mocioiu, M. Pospelov and R. Roiban, Phys. Lett. B 489, 390 (2000).

[11] N. Chair and M. M. Sheikh-Jabbari, Phys. Lett. B 504, 141 (2001).

[12] M. Chaichian, M. M. Sheikh-Jabbari and A. Tureanu, Phys. Rev. Lett. 86, 2716 (2001).

[13] J. E. Moyal, Proc. Cambridge Phil. Soc. 45, 99 (1947).

[14] M. Hayakawa, Phys. Lett. B 478, 394 (2000).

[15] S. Minwalla, M. Van Raamsdonk and N. Seiberg, JHEP 0002, 020 (2000).

[16] R. Szabo, Phys. Rept. 378, 207 (2003).

[17] A. Matusis, L. Susskind and N. Toumbas, JHEP 0012, 002 (2000).

[18] H. Arfaei and M. H. Yavartanoo, Phys. Lett. B 489, 390 (2000).

[19] I. F. Riad and M. M. Sheikh-Jabbari, JHEP 08, 045 (2000).

[20] F. Ardalan and N. Sadooghi, Int. J. Mod. Phys. A 16, 3151 (2001).

[21] N. Seiberg and E. Witten, JHEP 9909, 032 (1999).

[22] M. R. Douglas and N. Nekrasov, Rev. Mod. Phys. 73, 977 (2001).

[23] Z. Guralnik, R. Jackiw, S.Y. Pi and A.P. Polychronakos, Phys. Lett. B 517, 450 (2001). 
[24] Rong-Gen Cai, Phys. Lett. B 517, 457 (2001).

[25] S. M. Carroll, J. A. Harvey, V. A. Kostelecky, C. D. Lane and T. Okamoto, Phys. Rev. Lett. 87, 141601 (2001).

[26] A. A. Bichl, J. M. Grimstrup, L. Popp, M. Schweda and R. Wulkenhaar, hep-th/0102103 (2001).

[27] J. Lukierski, P. C. Stichel and W. J. Zakrzewski, Annals Phys. 260, 224 (1997).

[28] C. Duval and P. A. Horvathy, Phys. Lett. B 479, 284 (2000).

[29] P. A. Horvathy, Acta Phys. Polon. B 34, 2611 (2003).

[30] P. A. Horvathy and M. S. Plyushchay, JHEP 06, 033 (2002).

[31] P. A. Horvathy and M. S. Plyushchay, Phys. Lett. B 595, 547 (2004).

[32] P. A. Horvathy and M. S. Plyushchay, Nucl. Phys. B 714, 269 (2005).

[33] L. L. Foldy and S. A. Wouthuysen, Phys. Rev. 78, 29 (1950).

[34] Schweber S.S. An introduction to relativistic quantum field theory. Row, Peterson and Co., Evanston Ill., Elmsford N.Y., 1961.

[35] V. G. Bagrov, D. M. Gitman, M. C. Baldiotti and A. D. Levin, Ann. Phys. 14, 764 (2005).

[36] F. A. Berezin and M. S. Marinov, Ann. Phys. 104, 336 (1977).

[37] L. Brink, S. Deser, B. Zumino, P. di Vecchia and P. Howe, Phys. Lett. B 64 (1976) 435; L. Brink, P. di Vecchia and P. Howe, Nucl. Phys. B 118 (1977) 76; R. Casalbuoni, Nuovo Cimento A 33 (1976) 115; 389; A. Barducci, R. Casalbuoni and L. Lusanna, Nuovo Cimento A 35 (1976) 377; A.P. Balachandran, P. Salomonson, B. Skagerstam and J. Winnberg, Phys. Rev. D 15 (1977) 2308; D.M. Gitman, Nucl. Phys. B 488 (1997) 490

[38] D. M. Gitman and I. V. Tyutin, Quantization of Fields with Constraints, (SpringerVerlag, 1990).

[39] J.-M. Lévy-Leblond, Comm. Math. Phys. 6, 286 (1967).

[40] M. Chaichian, P. Kulish, K. Nishijima and A. Tureanu, Phys. Lett. B 604, 98 (2004). 
[41] V. G. Bagrov, M. C. Baldiotti, D. M. Gitman and A. D. Levin, Ann. Phys. 16, 274 (2007).

[42] M. C. Baldiotti and D. M. Gitman, Ann. Phys. 17, 450 (2008). 